\title{
Influence of rotating resonant magnetic perturbations on particle confinement
}

Qiming $\mathrm{Hu}^{1}$, Q. $\mathrm{Yu}^{2,}{ }^{2}$, Nengchao Wang ${ }^{1}$, Peng Shi ${ }^{1}$, Bing $\mathrm{Yi}^{1}$, Yonghua Ding ${ }^{1}$, Bo Rao ${ }^{1}$, Zhipeng

Chen ${ }^{1}$, Li Gao ${ }^{1}$, Xiwei $\mathrm{Hu}^{1}$, Hai Jin ${ }^{1}$, Mao $\mathrm{Li}^{1}$, Jianchao $\mathrm{Li}^{1}$, Kexun $\mathrm{Yu}^{1}$, Ge Zhuang ${ }^{1}$ and the

J-TEXT Team

${ }^{1}$ State Key Laboratory of Advanced Electromagnetic Engineering and Technology, School of Electric and Electronic Engineering, Huazhong University of Science and Technology, 430074, Wuhan,

China

${ }^{2}$ Max-Planck-Institut für Plasmaphysik, 85748 Garching, Germany

"Corresponding author: qiy@ipp.mpg.de

Abstract: The effect of resonant magnetic perturbations (RMPs) on particle confinement is studied in J-TEXT tokamak by using externally applied rotating RMPs. It is found that RMPs cause improved (degraded) particle confinement when its frequency is higher (lower) than the natural $m / n=2 / 1$ tearing mode frequency, and the amount of change in electron density is proportional to the difference between these two frequencies, where $m$ and $n$ are the poloidal and toroidal mode number, respectively. These results reveal the important role of the relative rotation between RMPs and the electron fluid in affecting the particle confinement. The experimental results are compared to numerical ones based on nonlinear two-fluid equations, and quantitative agreement is found.

Resonant magnetic perturbations (RMPs) often exist, e.g., in solar flares, magnetotail and fusion devices, due to intrinsic plasma instabilities and have attracted much research efforts, since they are associated with magnetic reconnection. In fusion devices RMPs can also be generated by external coil current, which have important applications in fusion plasmas. Static RMPs are able to suppress or mitigate edge localized modes (ELMs) [1-5] and to affect other instabilities [6-12]. In addition, rotating RMPs were used to study the field penetration [13], their effect on plasma rotation [14] and plasma response on TEXTOR [15]. On DIII-D, rotating RMPs are utilized to control NTMs rotation [16] and detect the intrinsic error field [17].

In tokamak experiments, static RMPs are often found to affect the electron density, either degrading or improving the particle confinement, e.g., on Tore Supra $[18,19]$ and TEXTOR [20, 21]. The improved particle confinement was achieved on TEXTOR when static RMPs is applied to the plasmas with neutral beam injection in the plasma current direction [20]. In J-TEXT Ohmic discharges in which $m / n=2 / 1$ modes rotate in the counter-current direction, degraded particle confinement is usually observed after applying static RMPs dominated by the 2/1 component [22], where $m$ and $n$ are the poloidal and toroidal mode number.

Up to now, however, there is no clear understanding about these different experimental phenomena and the underlying mechanism. It has been conjectured that the change in the particle confinement might be due to the formation of stochastic magnetic fields in the tokamak edge region caused by RMPs [23]. However, because of the relative rotation between the electron fluid and RMPs, usually there is little field stochasticity inside the separatrix due to the strong plasma screening effect. Based on the plasma response to RMPs in the framework of two-fluid equations, it has been shown theoretically that RMPs can affect particle confinement by changing the electron density gradient at corresponding resonant surfaces, so that tearing modes (TMs) are forced to take the same frequency as that of RMPs. Such a mechanism predicts that the change in particle confinement depends on the relative frequency between RMPs and the electron fluid [24, 25].

The purpose of the present study is to experimentally identify whether the relative rotation between RMPs and the electron fluid affects the results. Recently J-TEXT had been equipped with 12 
in-vessel saddle coils located at four toroidal locations [26], which can generate rotating RMPs with a maximum frequency up to $6 \mathrm{kHz}$ by supplying these coils with ac currents. This allows RMPs to rotate even faster than the 2/1 TM. It turns out that the change in the particle confinement is proportional to the frequency difference between the resonant mode and RMPs, as predicted in Ref. [24].

For the J-TEXT discharges only RMP components having the same sign for $m$ and $n$ are possibly resonant with plasmas. For our experiments with rotating RMPs, the amplitudes of these components with $m \times n>0$ at the plasma edge are plotted in Fig. 1(a), where the magnetic field is calculated for a frequency of $5 \mathrm{kHz}$, taking the eddy current in the vacuum vessel into account. There is a large $m / n=$ $2 / 1$ resonant component rotating in the direction opposite to the plasma current, $I_{\mathrm{p}}$. In addition, a large $2 / 3$ component also exists, but it has no corresponding resonant surface inside the plasma. All other components are much smaller. The amplitude of $2 / 1$ component at the plasma edge and at the $q=2$ surface (at about $r=20 \mathrm{~cm}$ ) are shown in Fig. 1(b) as a function of the RMP frequency. The eddy current in the vacuum vessel decreases the field amplitude to about 0.47 Gauss/kA at plasma edge $(0.36$ at $r=20 \mathrm{~cm})$ for $f_{\mathrm{RMP}}=5 \mathrm{kHz}$.
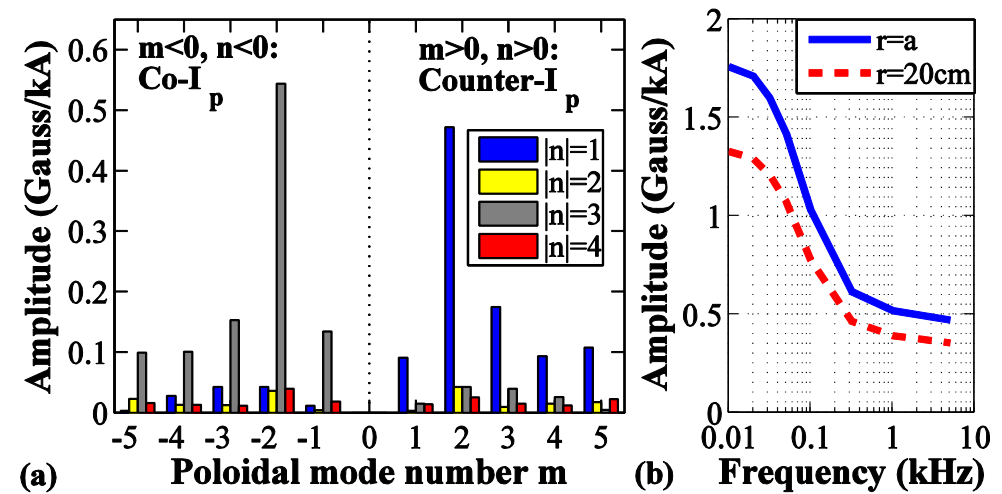

Figure. 1 (a) Spectrum of the radial magnetic field $b_{\mathrm{r}}$ generated by the RMP coils for $f_{\mathrm{RMP}}=5 \mathrm{kHz}$ at the plasma edge ( $r=a=26 \mathrm{~cm}$ ) with poloidal mode number $(|m|)$ in the range of $1-5$ and toroidal mode number $(|n|)$ in the range of 1-4. The field is calculated in vacuum condition. (b) The amplitude of the $m / n=2 / 1$ magnetic perturbation as a function of its frequency at $r=a$ and $20 \mathrm{~cm}$ (2/1 rational surface).

Our experiments have the following parameters: $I_{\mathrm{p}}=160 \mathrm{kA}$, the toroidal field $B_{\mathrm{t}}=1.55 \mathrm{~T}$, the aspect ratio $R_{0} / a=1.05 \mathrm{~m} / 0.26 \mathrm{~m}$, and the edge safety factor $q_{a}=3.15$. The saddle coil current $I_{\mathrm{c}}$ is applied during the stationary phase of Ohmic discharges. For all cases reported in this paper, an $m / n=$ 2/1 TM grows and saturates before the application of RMPs, and this mode rotates in the counter- $I_{\mathrm{p}}$ direction with a frequency $3.5-4.5 \mathrm{kHz}$. The $q=2$ rational surface is at about $1.05 \pm(0.18 \sim 0.2) \mathrm{m}$, obtained from two neighboring electron cyclotron emission (ECE) channels fluctuating out of phase with each other. The line-averaged plasma density $\bar{n}_{e}$ is in the range $(0.9-1.2) \times 10^{19} \mathrm{~m}^{-3}$, measured by the seven-channel far-infrared (FIR) $\mathrm{HCN}$ interferometer [27] at $R=1.0,1.05,1.1,1.15,1.2,1.25$ and $1.3 \mathrm{~m}$. The electron temperature is measured by the ECE radiometer without calibration.

An example of the effect of RMP on particle transport is shown in Fig. 2 with $f_{\mathrm{RMP}}=6 \mathrm{kHz}$ and $I_{\mathrm{c}}$ $=2.6 \mathrm{kA}$ at the flattop. Before applying RMPs, a $2 / 1 \mathrm{TM}$ saturates and rotates at a frequency $f_{2 / 1}=3.5$ $\mathrm{kHz}$ (Fig. 2(f)). At $t=0.225 \mathrm{~s}$, RMPs are turned on, and mode locking happens later at $t=0.24 \mathrm{~s}$, so that the $2 / 1 \mathrm{TM}$ is driven to rotate synchronously with RMPs at $6 \mathrm{kHz}$. The amplitude of the poloidal field perturbation $\tilde{B}_{\theta}$ becomes larger after mode locking (Fig. 2(e)), indicating a larger island width. The line-averaged electron density at $R=1.05 \mathrm{~m}$ (plasma core) increases from 1.08 to $1.22 \times 10^{19} \mathrm{~m}^{-3}$, 
with a relative change $\Delta \bar{n}_{e} / \bar{n}_{e 0}=13 \%$, where $\bar{n}_{e 0}$ is the initial electron density before the application of RMP, $\Delta \bar{n}_{e}=\bar{n}_{e}-\bar{n}_{e 0}$, and $\bar{n}_{e}$ is the density in steady state during applying RMPs. The density at plasma edge changes little as indicated by the time trace of $\bar{n}_{e}$ at $R=1.25 \mathrm{~m}$. The electron temperature decreases as shown by the ECE signal at $R=0.95 \mathrm{~m}$. The edge recycling decreases a little as indicated by the H $\alpha$ signal at $r=0.27 \mathrm{~m}$. At $t=0.355 \mathrm{~s}$ during $I_{\mathrm{c}}$ ramping down, the TM unlocks from the RMP. At the same time $\bar{n}_{e}$ begins to decrease, and the $2 / 1 \mathrm{TM}$ frequency slows down to $3.8 \mathrm{kHz}$. Results shown in Fig. 2 indicate that RMPs improves particle confinement in this case, and such an improvement mostly happens in the core region inside $q=2$ surface.

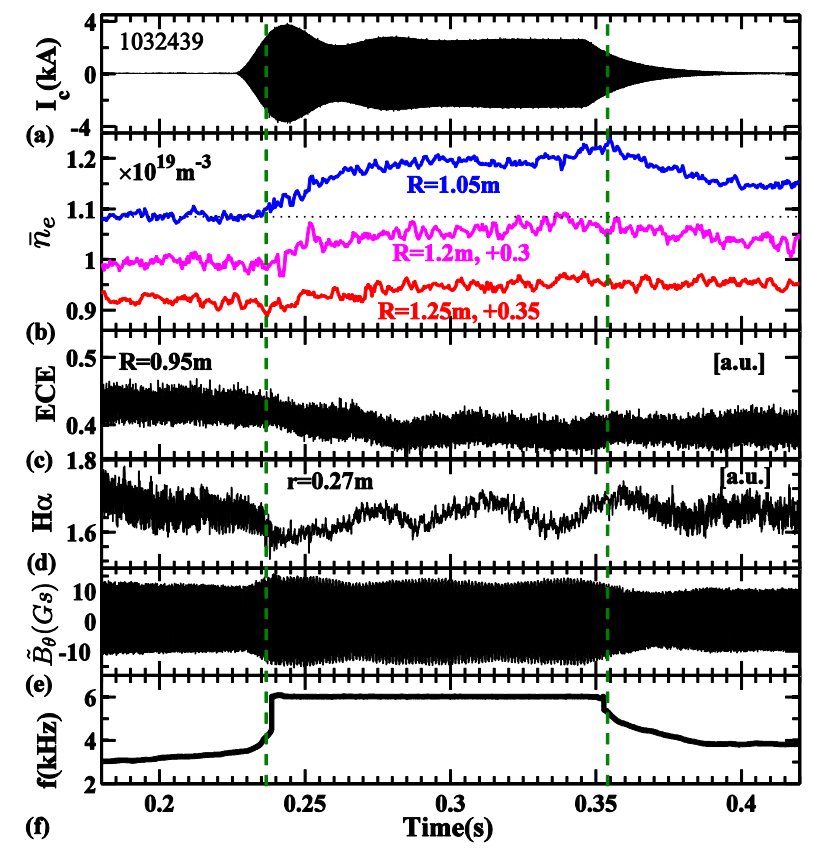

Figure. 2 Time traces of (a) RMP coil current $I_{\mathrm{c}}$, (b) line averaged density $\bar{n}_{e}$ at $R=1.05 \mathrm{~m}, 1.2 \mathrm{~m}$ and $1.25 \mathrm{~m}$, (c) ECE signal at $R=0.95 \mathrm{~m}$, (d) H $\alpha$ signal at $r=0.27 \mathrm{~m}$, (e) poloidal field perturbations $\tilde{B}_{\theta}$, and (f) $2 / 1 \mathrm{TM}$ frequency $f_{2 / 1}$. The RMP frequency is $6 \mathrm{kHz}$. For comparison, $\bar{n}_{e}$ is added by 0.3 and 0.35 for the channels at $R=1.2$ and 1.25 $\mathrm{m}$.

For the results shown in Fig. 2, the initial frequency difference between the RMP and TM is $\Delta f=$ $f_{\mathrm{RMP}}-f_{2 / 1}=2.5 \mathrm{kHz}$. Fig. 3 shows the results from other two shots for $f_{2 / 1}=4 \mathrm{kHz}$ and $I_{\mathrm{c}}=2.5 \mathrm{kA}$ at the flattop, with $f_{\mathrm{RMP}}=4.85 \mathrm{kHz}(\Delta f=0.85 \mathrm{kHz}$, shot 1032451$)$ and $2 \mathrm{kHz}(\Delta f=-2 \mathrm{kHz}$, shot 1032082$)$, respectively. For $\Delta f=0.85 \mathrm{kHz}$, during the mode locking $\bar{n}_{e}$ slightly increases with $\Delta \bar{n}_{e} / \bar{n}_{e 0}=3.5 \%$. The edge recycling decreases a little, and the island width slightly increases, as indicated by the $\mathrm{H} \alpha$ and $\tilde{B}_{\theta}$ signals. While for $\Delta f=-2 \mathrm{kHz}, \bar{n}_{e}$ decreases with $\Delta \bar{n}_{e} / \bar{n}_{e 0}=-9.5 \%$, and both the edge recycling and island width increase. 


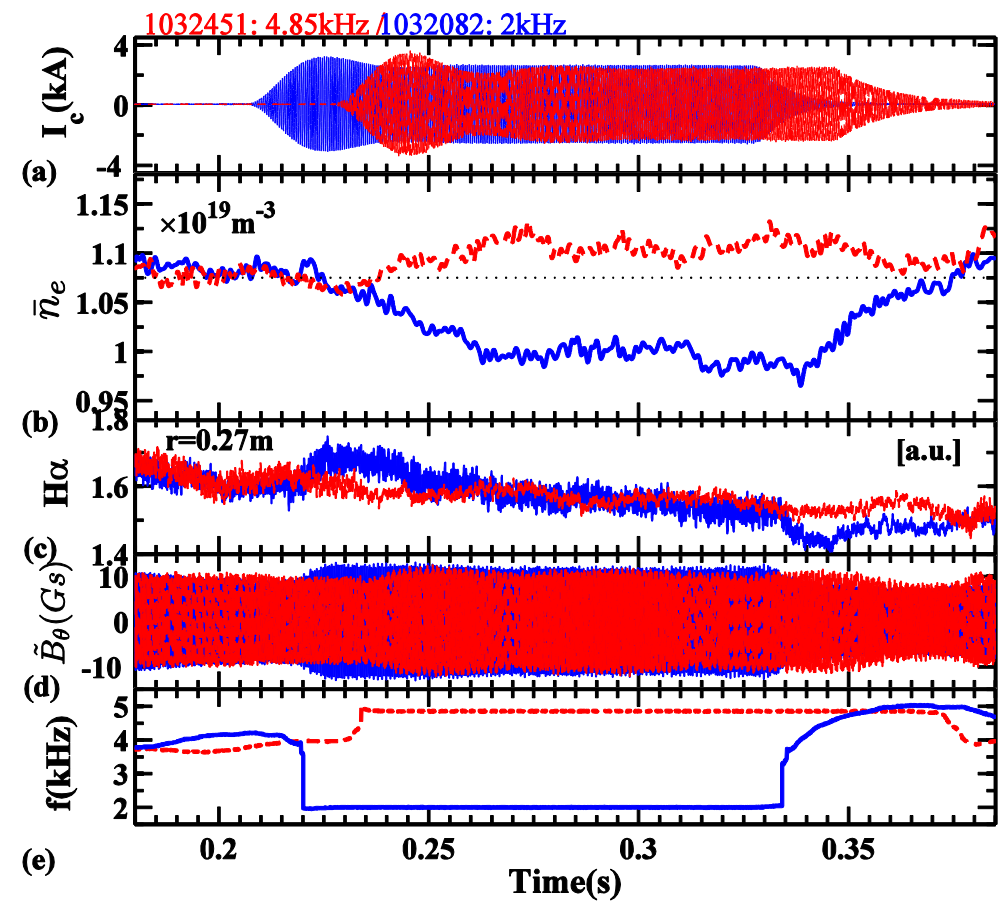

Figure. 3 Time traces of (a) RMP coil current $I_{\mathrm{c}}$, (b) line averaged density $\bar{n}_{e}$ at $R=1.05 \mathrm{~m}$, (c) H $\alpha$ signal at $r=0.27$ m, (d) poloidal field perturbations $\tilde{B}_{\theta}$ and (e) $2 / 1 \mathrm{TM}$ frequency $f_{2 / 1}$. Here, $f_{\mathrm{RMP}}$ equals $4.85 \mathrm{kHz}$ and $2 \mathrm{kHz}$ for shots 1032451 and 1032082 , respectively.

In addition to the value of $\Delta f$, the RMP amplitude also matters. Fig. 4(a) shows the cases for $\Delta f \sim$ $2 \mathrm{kHz}\left(f_{\mathrm{RMP}}=6 \mathrm{kHz}\right.$ and $\left.f_{2 / 1} \sim 4 \mathrm{kHz}\right)$ with $I_{\mathrm{c}}=1.4,1.8$, and $2.5 \mathrm{kA}$. For $I_{\mathrm{c}}=1.4 \mathrm{kA}$, the RMP is not strong enough to cause mode locking, and no obvious change in $\bar{n}_{e}$ has been observed. For $I_{\mathrm{c}}=1.8$ and $2.5 \mathrm{kA}$, mode locking happens, and a stronger RMP causes a larger increase in $\bar{n}_{e}$. Fig. 4(b) shows $\Delta \bar{n}_{e} / \bar{n}_{e 0}$ versus $I_{\mathrm{c}}$ for $\Delta f=2 \pm 0.2 \mathrm{kHz}$. A larger $I_{\mathrm{c}}$ causes a bigger change in the density for $I_{\mathrm{c}}<$ $2.8 \mathrm{kA}$, but the saturation in $\Delta \bar{n}_{e} / \bar{n}_{e 0}$ appears for $I_{\mathrm{c}} \geq 2.8 \mathrm{kA}$. Such saturation is also seen in nonlinear numerical results for sufficiently strong RMPs [24]. Results shown in Fig. 4(c) and 4(d) are for $\Delta f \sim-2$ $\mathrm{kHz}$ with different RMP amplitude. In this case a stronger RMP causes a larger decrease in $\bar{n}_{e}$ for 1.6 $\mathrm{kA}<I_{\mathrm{c}}<2.9 \mathrm{kA}$.
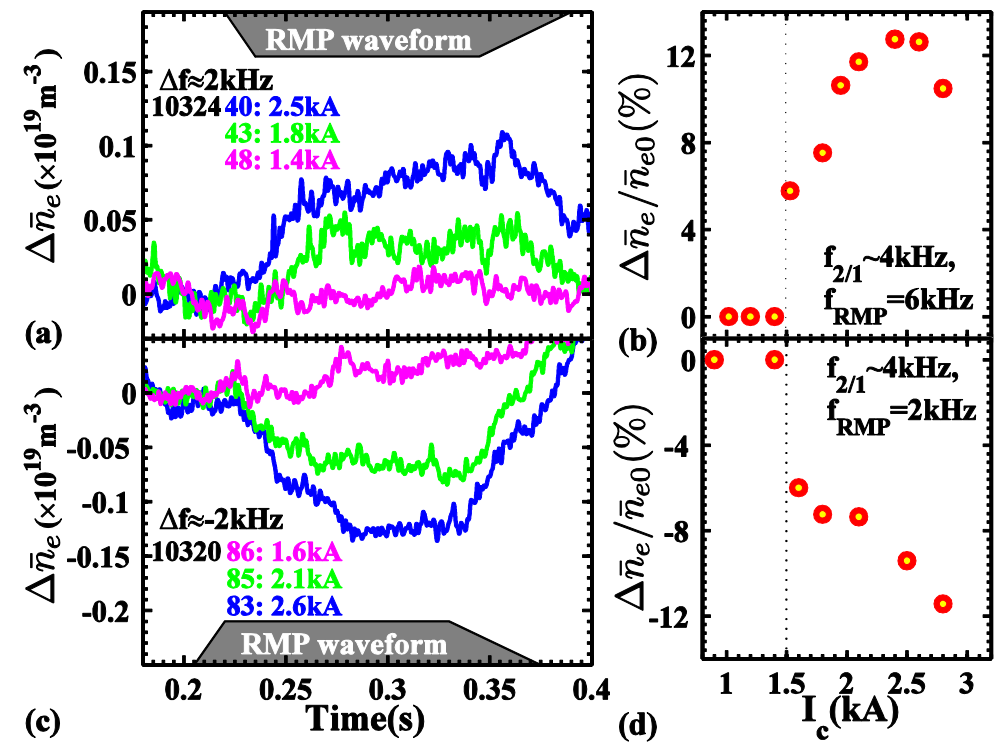
Figure. 4 (a) Time traces of the change in line averaged density $\Delta \bar{n}_{e}$ at $R=1.05 \mathrm{~m}$ for different coil current $I_{\mathrm{c}}$ with $\Delta f \sim 2 \mathrm{kHz}$. (b) $\Delta \bar{n}_{e} / \bar{n}_{e 0}$ versus $I_{\mathrm{c}}$ with $\Delta f=2 \pm 0.2 \mathrm{kHz}$. (c) Similar to (a) but with $\Delta f=-2 \pm 0.2 \mathrm{kHz}$. (d) $\Delta \bar{n}_{e} / \bar{n}_{e 0}$ versus $I_{\mathrm{c}}$ with $\Delta f=-2 \pm 0.2 \mathrm{kHz}$.

Systematical experiments have been carried out by keeping the background plasma parameters to be the same, while only the amplitude and frequency of RMPs are changed: $f_{\mathrm{RMP}}=1,2,3,4,4.85$ and $6 \mathrm{kHz}$, and for each frequency $I_{\mathrm{c}}$ is scanned from $1 \mathrm{kA}$ to $3.2 \mathrm{kA}$. The mode locking usually happens in experiments for $I_{\mathrm{c}}>1.6 \mathrm{kA}$. In Fig. 5(a), $\Delta \bar{n}_{e} / \bar{n}_{e 0}$ is shown as a function of $\Delta f\left(=f_{\mathrm{RMP}}-f_{2 / 1}\right) . \Delta \bar{n}_{e}$ is the change in electron density at plasma core in steady state by RMPs with $1.6 \mathrm{kA}<I_{\mathrm{c}}<3.2 \mathrm{kA}$ (squares). The results with about the same value of $I_{\mathrm{c}}, I_{\mathrm{c}}=2.4 \pm 0.2 \mathrm{kA}$, are marked by triangles. It is seen that RMPs improve particle confinement $\left(\Delta \bar{n}_{e} / \bar{n}_{e 0}>0\right)$ for $\Delta f>0$ but degrade it for $\Delta f<0$, and $\Delta \bar{n}_{e} / \bar{n}_{e 0}$ linearly depends on $\Delta f$ if the RMP amplitude is about the same. The edge recycling is found to decrease for $\Delta \bar{n}_{e} / \bar{n}_{e 0}>0(\Delta f>0)$ but increase for $\Delta \bar{n}_{e} / \bar{n}_{e 0}<0(\Delta f<0)$. Fig. 5(a) indicates the significant role of the relative frequency $\Delta f$ in the changed particle confinement by RMPs. The results obtained by static RMPs are also shown in Fig. 5(a) by circles. A linear dependence of $\Delta \bar{n}_{e} / \bar{n}_{e 0}$ on $\Delta f$ exists when including the results from both the static and rotating RMPs.

Fig. 5(b) shows $w / w_{0}$ as a function of $\Delta f$, where $w / w_{0} \approx\left(\tilde{B}_{\theta} / \tilde{B}_{\theta 0}\right)^{0.5}$, and $w_{0}(w)$ and $\tilde{B}_{\theta 0}\left(\tilde{B}_{\theta}\right)$ are the island width and poloidal field perturbation amplitude before (after) applying rotating RMPs. The island width is increased for both $\Delta f>0$ and $\Delta f<0$ (w/w $w_{0}>1.2$ for all cases), and it decrease with increasing $\Delta f$. Fig. 5(c) shows $\Delta \bar{n}_{e} / \bar{n}_{e 0}$ as a function of $w / w_{0}$. There is a trend that $\Delta \bar{n}_{e} / \bar{n}_{e 0}$ decreases with increasing $w / w_{0}$. The central electron temperature is, however, always found to decrease for both $\Delta f>0$ and $\Delta f<0$, as shown in Fig. 2, being consistent with the increased island width by RMPs and the fast parallel electron heat diffusion.
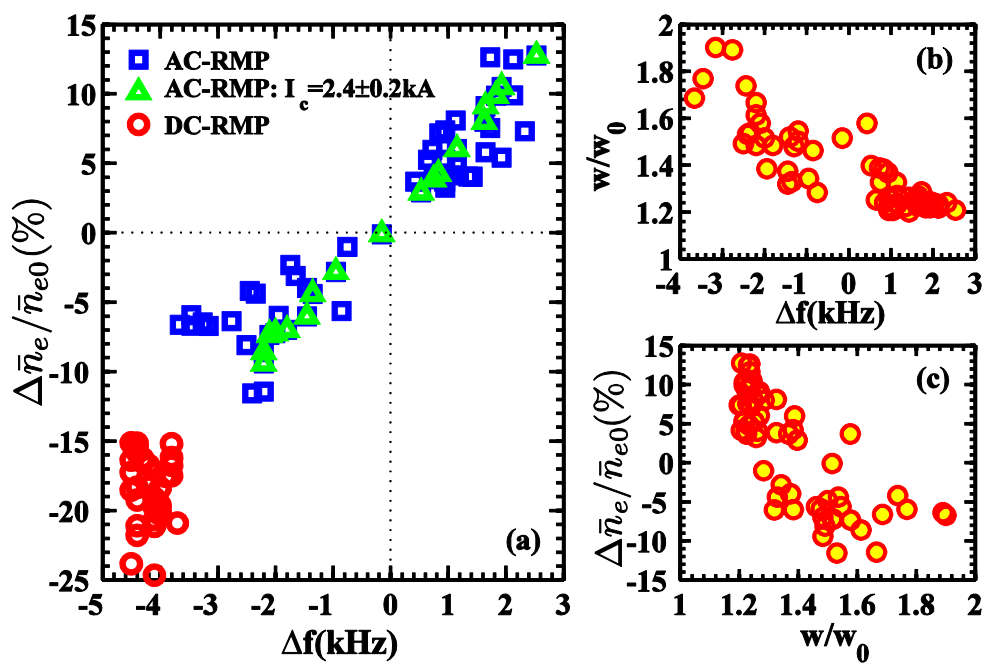

Figure. 5 (a) Relative change in line averaged density $\Delta \bar{n}_{e} / \bar{n}_{e 0}$ at plasma core versus the frequency difference $\Delta f$, (b) $w / w_{0}$ versus $\Delta f$ and (c) $\Delta \bar{n}_{e} / \bar{n}_{e 0}$ versus $w / w_{0}$, where $w_{0}$ and $w$ are the island width before and after applying RMPs, respectively. The TM frequency $f_{2 / 1}$ ranges from $3.5 \mathrm{kHz}$ to $4.5 \mathrm{kHz}$, and the RMP coil current $I_{\mathrm{c}}$ ranges from $1.6 \mathrm{kA}$ 
to $3.2 \mathrm{kA}$. A linear relation between $\Delta \bar{n}_{e} / \bar{n}_{e 0}$ and $\Delta f$ exists for about the same value of $I_{\mathrm{c}}$ (triangles).

The intrinsic $2 / 1$ error field on J-TEXT is measured to be $0.3 \mathrm{G}$ at the plasma edge $r=a$ [28], being weaker than rotating RMPs (a few Gausses). The effect of rotating RMPs on the electron density is observed so far only when there is an $m / n=2 / 1 \mathrm{TM}$ in discharges. For tearing-stable plasmas, rotating RMP has been applied with $I_{\mathrm{c}}$ up to $3.5 \mathrm{kA}$, but neither mode penetration nor obvious change in electron density was observed. Together with the results shown in Figs. 2 and 5, the dependence of $\Delta \bar{n}_{e}$ on the radial location and $\Delta f$, it indicates that the observed effect is a resonant one. In previous J-TEXT experiments using static RMPs, the edge safety factor $q_{\mathrm{a}}$ was varied from 2.7 to 3.8 , and RMPs were varied from a spectrum dominated by the $2 / 1$ component to others with negligible $2 / 1$ component. Significant change in the electron density is observed only when there was a large resonant 2/1 component RMP to cause mode locking or mode penetration [22]. The required static RMP amplitude at $r=a$ to cause mode locking is about the same as that of rotating RMPs if the value of $\Delta f$ is about the same [22].

The threshold for mode locking, being proportional to $\Delta f$ and inverse proportional to the mode amplitude [9], is lower than that for mode penetration. $|\Delta f|$ is smaller when RMPs rotate in the same direction as the mode, so that the required RMP amplitude to cause mode locking is lower than that of static RMPs or error field for which $\Delta f$ is larger.

When the RMP amplitude is not too large, the quasi-linear formula shows that the change in the local plasma density gradient by RMP is given by [24]

$$
r_{\mathrm{s}}\left(\Delta n_{\mathrm{e}}\right)^{\prime} / n_{\mathrm{e}}=-r_{\mathrm{s}}^{2} d_{1}\left(\omega-\omega_{E 0}-\omega_{* e 0}\right)\left|b_{1 \mathrm{r}} / B_{\mathrm{t}}\right|^{2} /\left(m D_{\perp}\right),
$$

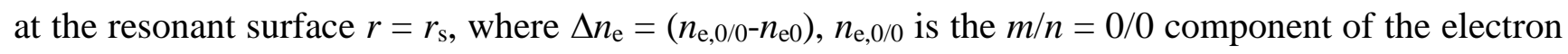
density $n_{\mathrm{e}}$ after applying RMPs, $n_{\mathrm{e} 0}$ is the original density unperturbed by the RMP, and $b_{1 r}$ is the radial magnetic field perturbation. Here, $d_{1}$ is the ratio between the electron cyclotron frequency and the collisional frequency, $D_{\perp}$ is the perpendicular particle diffusivity, and $\omega, \omega_{\mathrm{E} 0}$ and $\omega_{* \mathrm{e} 0}$ are the angular frequency of the RMP, electron electric and diamagnetic drifts, respectively. As the 2/1 TM frequency $f_{2 / 1}=f_{\mathrm{E} 0}+f_{* \mathrm{e} 0}$ [8], equation (1) can be written into another form,

$$
r_{\mathrm{s}}\left(\Delta n_{\mathrm{e}}\right)^{\prime} / n_{\mathrm{e}}=-2 \pi r_{\mathrm{s}}^{2} d_{1}\left(f_{R M P}-f_{2 / 1}\right)\left|b_{1 \mathrm{r}} / B_{\mathrm{t}}\right|^{2} /\left(m D_{\perp}\right) .
$$

Equation (2) indicates that the local density gradient decreases $\left(\left|n_{\mathrm{e}}{ }^{\prime} / n_{\mathrm{e}}\right|\right.$ increases in the standard case where the equilibrium density gradient is negative) for $f_{\mathrm{RMP}}>f_{2 / 1}$, while in the opposite limit $\left(\Delta n_{\mathrm{e}}\right)^{\prime}>0$. Therefore, the electron density increases for $f_{\mathrm{RMP}}>f_{2 / 1}$ but decreases for $f_{\mathrm{RMP}}<f_{2 / 1}$, being consistent with the experimental results shown in Fig. 5.

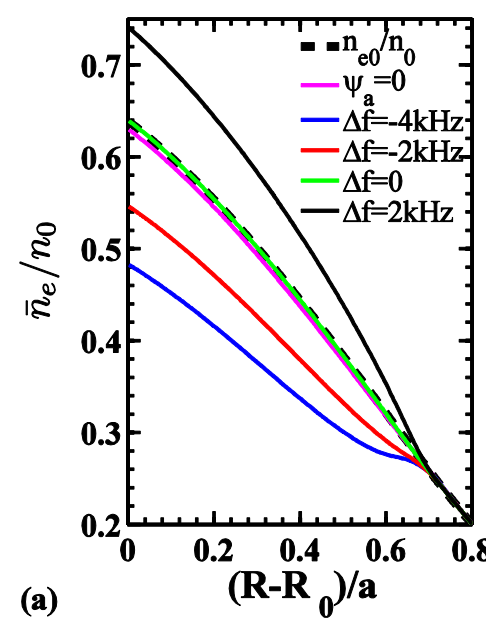

(a)

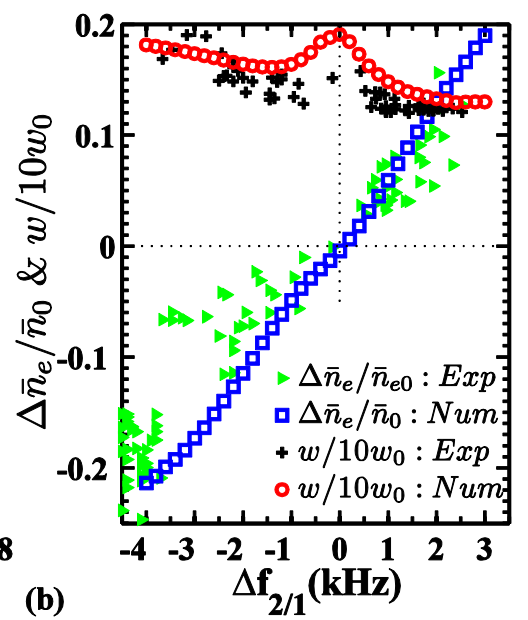

(b) 
Figure. 6 Numerical modeling results are shown. (a) Radial profiles of line-averaged density $\bar{n}_{e} / n_{0}$ for $\Delta f=-4,-2,0$ and $2 \mathrm{kHz}\left(f_{\mathrm{RMP}}=0,2,4,6 \mathrm{kHz}\right)$ in steady state. The RMP amplitude is $\psi_{a}=6 \times 10^{-5}\left(b_{1 r^{2}, 1}(a)=1.85 \mathrm{Gauss}\right)$, and $f_{2 / 1}$ $=4 \mathrm{kHz}$. The line-average is taken at a constant $R$ value, and $R_{0}$ is the major radius of the plasma center. (b) Relative change in line averaged density $\Delta \bar{n}_{e} / \bar{n}_{0}$ (blue squares) at plasma core and the relative island width $w / w_{0}$ (red circles) versus $\Delta f$. Experimental results are also shown by triangles $\left(\Delta \bar{n}_{e} / \bar{n}_{0}\right)$ and crosses $\left(w / w_{0}\right)$ for comparison.

Eq. (2) is subjected to the quasi-linear assumption and only gives a qualitative understanding. To compare with experimental results, full nonlinear numerical calculations have been carried out based on two-fluid equations, including the mass conservation equation, the generalized Ohm's law and the equation of motion in the perpendicular and parallel (to magnetic field) directions, as described in Ref. [24]. The input parameters are based on experimental values mentioned above. A monotonic $q$ profile is used with the $q=2$ surface located at $r_{\mathrm{s}}=0.7 a$. The $2 / 1 \mathrm{TM}$ is unstable without applying RMP. At the $q=2$ surface, $n_{\mathrm{e}}=0.7 \times 10^{19} \mathrm{~m}^{-3}$ and electron temperature $T_{\mathrm{e}}=300 \mathrm{eV}$. The plasma viscosity and perpendicular particle diffusivity are assumed to be $\mu=5 D_{\perp}=0.5 \mathrm{~m}^{2} / \mathrm{s}$. A momentum source is included in the equation of motion to drive the plasma rotation, so that the $2 / 1$ mode rotates in the electron diamagnetic drift direction with a frequency $f_{2 / 1}=4 \mathrm{kHz}$. At nonlinear saturation the island has a width $w_{0}=0.082 \mathrm{a}$, causing a slight decrease in electron density as shown in Fig. 6(a). The radial profile of original equilibrium line-averaged density profile $\bar{n}_{e 0} / n_{0}$ is also shown there, where $n_{0}$ is $n_{\mathrm{e} 0}(r=0)$.

After applying rotating RMPs with $\psi_{\mathrm{a}}=6 \times 10^{-5}\left(b_{1 \mathrm{r}}^{2,1}(a)=1.85\right.$ Gauss $)$, radial profiles of $\mathrm{m} / \mathrm{n}=$ 0/0 component of the line-averaged electron density, $\bar{n}_{e} / n_{0}$ (normalized to $n_{0}$ ), in steady state are shown in Fig. 6(a) for $f_{\mathrm{RMP}}=0,2,4$ and $6 \mathrm{kHz}$ (i.e. $\Delta f=-4,-2,0$ and $2 \mathrm{kHz}$ ). The line-averaged electron density decreases for $\Delta f=-4$ and $-2 \mathrm{kHz}$ but increases for $\Delta f=2 \mathrm{kHz}$ inside the $\mathrm{q}=2$ surface, and $\bar{n}_{e} / n_{0}$ changes little for $\Delta f=0$, which agree with the experimental results shown in Figs. 2-5. The change in electron density is larger for $\Delta f=-4 \mathrm{kHz}$ than that for $\Delta f=-2 \mathrm{kHz}$.

Fig. 6(b) shows the relative change in line-averaged electron density, $\Delta \bar{n}_{e} / \bar{n}_{0}$, and the saturated island width as a function of $\Delta f$. It is seen that $\Delta \bar{n}_{e} / \bar{n}_{0}$ linearly depends on $\Delta f$, and it is negative (positive) for $\Delta f<0(\Delta f>0)$. The experimental results are also shown in Fig. 6(b) by triangles. The numerical results approximately agree with experimental ones.

The change of the electron temperature has not been calculated here. However, based on previous calculation results [25], the electron temperature is expected to decrease with the experimental parameters as input.

In conclusion, both improvement and deterioration of particle confinement caused by rotating RMPs have been observed in J-TEXT discharges in the presence of the $m / n=2 / 1 \mathrm{TM}$. The applied rotating RMP causes confinement improvement (deterioration) when its frequency is higher (lower) than the natural frequency of the 2/1 TM, and the applied RMP causes more change in the electron density for a larger difference between these two frequencies. These results reveal the role of relative rotation between the electron fluid and RMPs in affecting the particle transport. Numerical results based on nonlinear two-fluid equations approximately agree with experimental ones.

The externally applied RMP leads to an electromagnetic force which depends on the relative 
rotation between the electron fluids and the RMP [29]. The change of the electron density gradient is required for achieving electron force balance [24]. It is interesting to notice that our result has an analogy to "rotating wall" technique previously used in non-neutral plasma experiments, in which the relative change of electron density is proportional to the difference between the applied perturbation frequency and the electron rotation [30-32]. In both cases the electron force balance is affected by the relative rotation between the electron fluid and external perturbation and therefore by the electron density.

Acknowledgement: This work is supported by the Ministry of Science and Technology (Contract No. 2011GB109001 and 2014GB118000) and the National Natural Science Foundation (Contract No. 10990214, 11275080 and 11105056) of China.

\section{References}

[1] T. E. Evans et al., Phys. Rev. Lett. 92, 235003 (2004).

[2] W. Suttrop et al., Phys. Rev. Lett. 106, 225004 (2011).

[3] Y. M. Jeon et al., Phys. Rev. Lett. 109, 035004 (2012).

[4] Y. Liang et al., Phys. Rev. Lett. 98, 265004 (2007).

[5] A. Kirk et al., Nucl. Fusion 50, 034008 (2010).

[6] Y. Q. Liu et al., Phys. Plasmas 7, 3681 (2000).

[7] S. A. Sabbagh et al., Phys. Rev. Lett. 97, 045004 (2006).

[8] T. C. Hender et al., Nucl. Fusion 32, 2091 (1992).

[9] R. Fitzpatrick, Nucl. Fusion 33, 1049 (1993).

[10] Q. Yu et al., Nucl. Fusion 40, 2031 (2000).

[11] Q. Hu et al., Nucl. Fusion 52, 083011 (2012).

[12] Q. Hu et al., Phys. Plasmas 20, 092502 (2013).

[13] Y. Kikuchi, et al., Phys. Rev. Lett. 97, 085003 (2006).

[14] M. F. M. De Bock, et al., Nucl. Fusion 48, 015007 (2008).

[15] Y. Yang, et al., Nucl. Fusion 52, 074014 (2012).

[16] F.A. Volpe, et al., Phys. Plasmas 16102502 (2009).

[17] D. Shiraki, et al., Nucl. Fusion 54, 033006 (2014).

[18] J. C. Vallet et al., Phys. Rev. Lett. 67, 2662 (1991).

[19] T. E. Evans et al., J. Nucl. Mater. 196-198, 421 (1992).

[20] K. H. Finken et al., Phys. Rev. Lett. 98, 065001 (2007).

[21] O. Schmitz et al., J. Nucl. Mater. 390-91, 330 (2009).

[22] Q. Hu et al., Nucl. Fusion 54, 064013 (2014).

[23] M. Z. Tokar et al., Phys. Rev. Lett. 98, 095001 (2007).

[24] Q. Yu et al., Nucl. Fusion 49, 062001 (2009).

[25] Q. Yu et al., Nucl. Fusion 51, 073030 (2011).

[26] B. Rao et al., Fusion Eng. Des. 89, 378-384 (2014).

[27] L. Gao et al., Rev. Sci. Instrum. 83, 10E303 (2012).

[28] B. Rao, et al., Rev. Sci. Instrum. 84, 043504 (2013).

[29] Q. Yu et al., Phys. Plasmas 16, 042301 (2009).

[30] J. R. Danielson, er al., Phys. Rev. Lett. 99, 135005 (2007).

[31] X. P. Huang, et al., Phys. Rev. Lett. 78, 875 (1997).

[32] E. M. Hollmann, et al., Phys. Plasmas 7, 2776 (2000). 